\title{
Oligomeric Fluorescent Labels for DNA
}

Andrea Cuppoletti, Younjin Cho, Jin-Seong Park, Christoph Strässler, Eric T. Kool*

Department of Chemistry, Stanford University, Stanford CA 94305-5080

*To whom correspomdence should be addressed. Fax: 650-725-0259, e-mail: kool@stanford.edu

\section{CONTENTS:}

Figure S1. Temperature effects on absorption for oligomeric labels

(p. 2)

Method sfor CD spectra

(p. 3)

Preliminary analysis of dye interactions

(p. 3)

Figure S2. CD spectra of oligomeric labels

Table S1. Excimer-to-monomer emission ratios for pyrene labels

Figure S3. Effect of ionic strength on emission of three trimeric fluorophores

Figure S4. Effect of $\mathrm{pH}$ on emission of three trimeric fluorophores

Figure S5. Time courses and spectra for photobleaching of trimeric fluorophores (p. 9) 

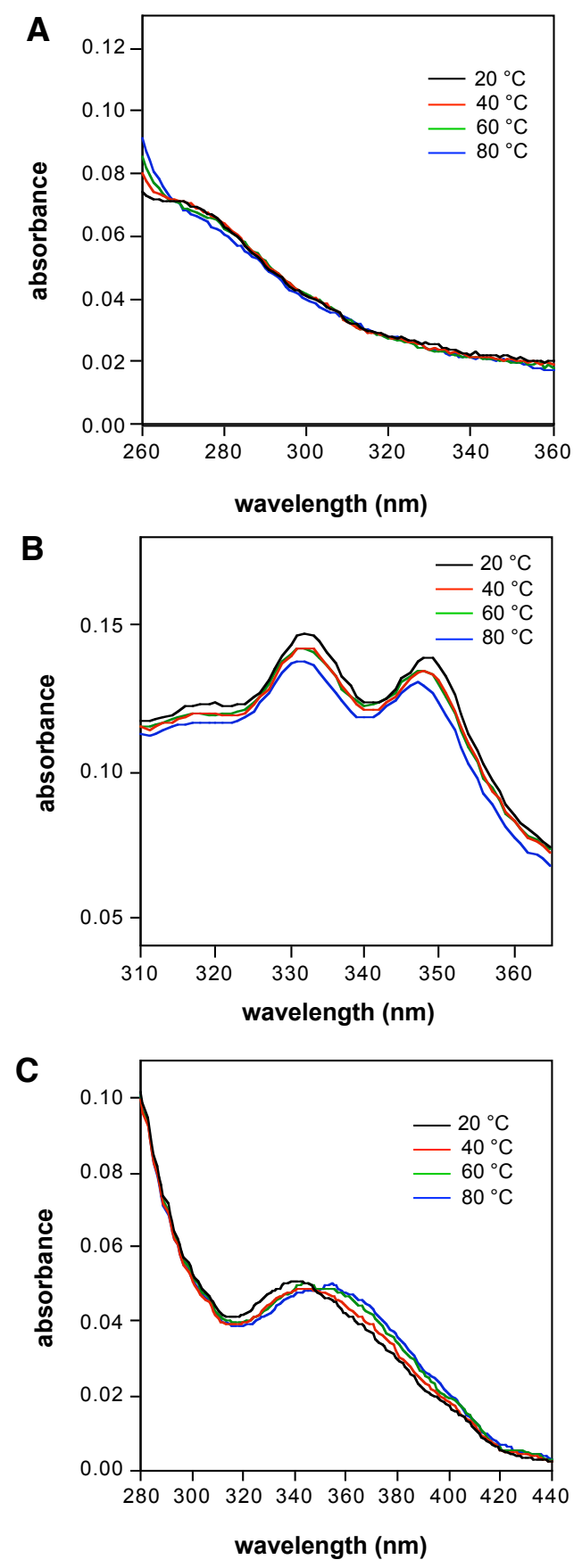

Figure S1. Temperature dependence of UV absorption for oligomeric labels conjugated to singlestranded DNA in TBE buffer (Table 1, note "a"). Shown are (terphenyl) 5 (A); (pyrene) 5 (B); and (terthiophene) ${ }_{5}(\mathrm{C})$. 
CD spectra methods. CD spectra were recorded on a JASCO 860 polarimeter. In a general experiment a quartz cuvette with $1 \mathrm{~cm}$ length path, equipped with a Teflon stopper, was charged with $1.0 \mathrm{~mL}$ of a solution of the oligonucleotide in TBE buffer $(\mathrm{pH}=7.0)$. All the spectra were recorded at room temperature, and corrected for background and solvent absorption.

Preliminary analysis of intrastrand dye interactions. Figure S2 shows the CD data for the three fluorescent deoxyriboside series. For the terphenyl series, the CD measurements reveal a strong exciton splitting with four and five monomers, a weaker effect with three, and little or no splitting with one and two chromophores. The positive exciton chirality suggests $(+)$ helical orientation of the chromophores' long axes relative to one another (refs. 30,31, 32 in main paper). Taken together with the significant red shift with increasing monomer number (from 264 to $273 \mathrm{~nm}$ ) in the absorption spectra (Figure 2, main paper) and the large red shift with increasing numbers of monomers (from 344 to $383 \mathrm{~nm}$ ) in the emission spectra (Figure 3), the experiments strongly point to intramolecular "J" aggregate behavior for this dye series $(9,10)$. (Note that "aggregate" here refers to intrastrand interaction of dyes, and not intermolecular aggregation.) This suggests an organized structure in which the long axes of the electric transition dipoles are parallel but partially oriented in head-to-tail fashion. Such an orientation might arise if dyes are all angled downward from a vertically oriented phosphodiester backbone, although other arrangements are also possible. Clearly there is also a positive helical twist in the aggregate, caused by the inherent chirality of the DNA backbone.

The terthiophene series shows similar behavior (Fig. S2,B), with clear evidence of exciton splitting with positive exciton chirality. However, one difference is that a maximum in the CD is seen at three dyes, and the magnitude diminishes somewhat with four and five dye monomers. Taken together with a marked blue shift in absorption with increasing numbers of monomers (from 373 to $342 \mathrm{~nm}$ ), along with a sharpening of the 0,0 band and the fluorescence quenching in the series (Table 1), we tentatively assign the terthiophene oligomers as "H" aggregates $(23,24,25)$,. This suggests a significant structural organization in the oligomers, such that their long axes are oriented in the same direction (roughly parallel). The strong quenching of the emission along with the small CD intensity suggests a small angle between the transition dipoles of the dyes, as it might occur if they lie face-to-face; such an orientation seems reasonable in the context of the repeating sequence of DNA if the backbone is oriented along one side and the dyes all are oriented away and in the same direction. The DNA backbone adds positive helical chirality to this aggregate.

The oligomeric pyrene series, displays different behavior in the CD spectra (Fig. S2C). We observe clear evidence of exciton splitting only for the case with two chromophores, and small or no Cotton 
effects for the case with three pyrenes. Interestingly, negative CD effects are seen for the cases with four and five pyrenes, with no bisignate pattern in the 1La transition absorption peak, which suggests that there is no exciton coupling. The absence of shifts in the absorption spectra also is consistent with the lack of exciton splitting (with the bis-pyrene case as an exception). As the number of monomers increases, the excimer to monomer ratio increases markedly, and the cases with four and five pyrenes show little remaining monomer emission. Excimer to monomer ratios are 0, 0.4, 0.9, 9.9, and 11.1 for one through five monomers (Table S1) (8). Overall, the data are consistent with an interpretation in which there is ground state aggregation among the pyrene dyes in the multi-pyrene oligomers. However, there is likely a change in orientation induced by addition of a third, fourth and fifth pyrene to the two-pyrene case. More data will be needed to interpret the structural differences in a convincing way. 

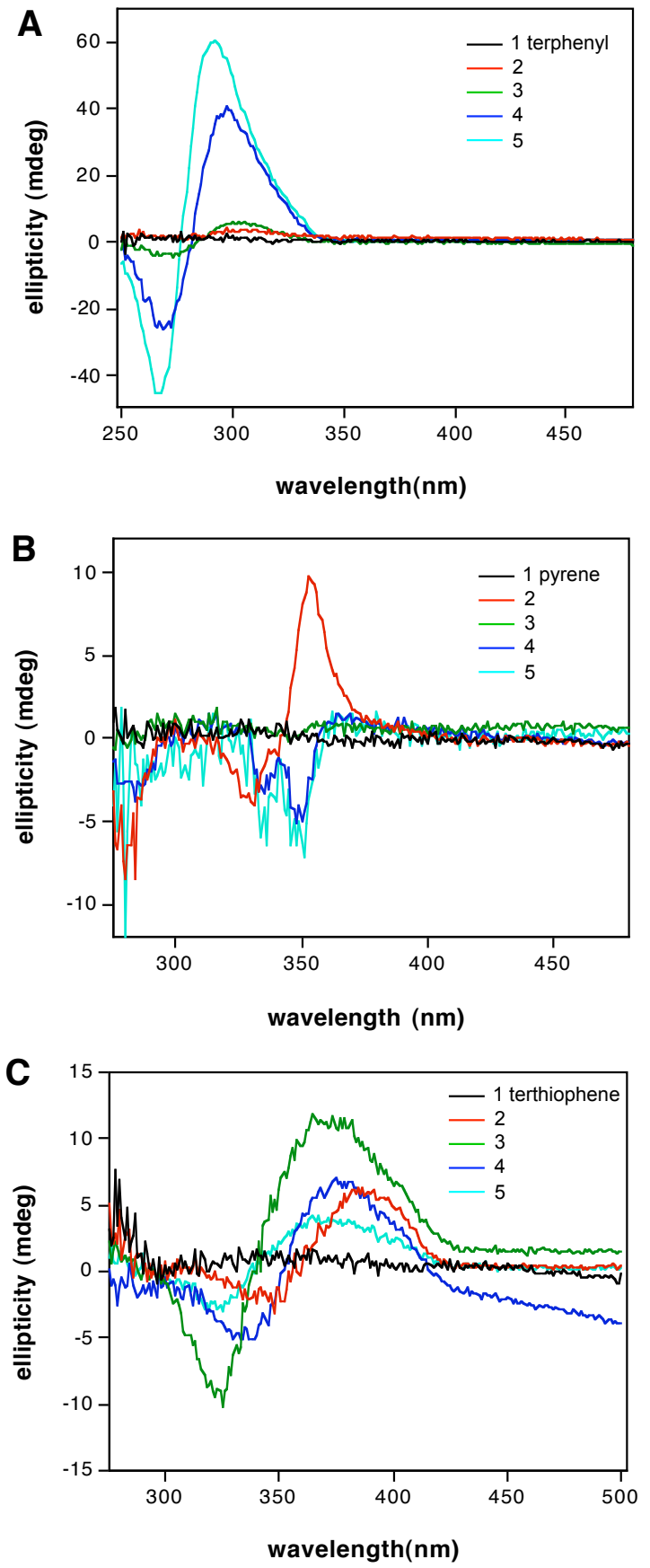

Figure S2. CD spectra for oligomeric terphenyl (a); oligomeric pyrene (b); and oligomeric terthiophene (c) labels conjugated to DNA. Data were taken in aqueous buffer (Table 1, note a). 
Table S1. Monomer-to-excimer ratios for pyrene deoxyriboside oligomers. ${ }^{a}$

\begin{tabular}{lccc}
\hline $\begin{array}{l}\text { fluorescent } \\
\text { species }\end{array}$ & $\begin{array}{l}\mathrm{I}_{\max } \\
(\text { monomer })\end{array}$ & $\begin{array}{l}\mathrm{I}_{\max } \\
(\text { excimer })\end{array}$ & $\begin{array}{l}\text { Intensity ratio } \\
\text { (excimer:monomer) }\end{array}$ \\
\hline pyrene & $6.2 \mathrm{E} 6$ & 0 & 0 \\
$(\text { pyrene })_{2}$ & $12.3 \mathrm{E} 6$ & $4.7 \mathrm{E} 6$ & 0.4 \\
$(\text { pyrene })_{3}$ & $7.4 \mathrm{E} 6$ & $6.7 \mathrm{E} 6$ & 0.9 \\
$(\text { pyrene })_{4}$ & $9.9 \mathrm{E} 5$ & $9.8 \mathrm{E} 6$ & 9.9 \\
$(\text { pyrene })_{5}$ & $9.3 \mathrm{E} 5$ & $10.4 \mathrm{E} 6$ & 11.1 \\
\hline
\end{tabular}

${ }^{a}$ Measured from peak area integrations. 

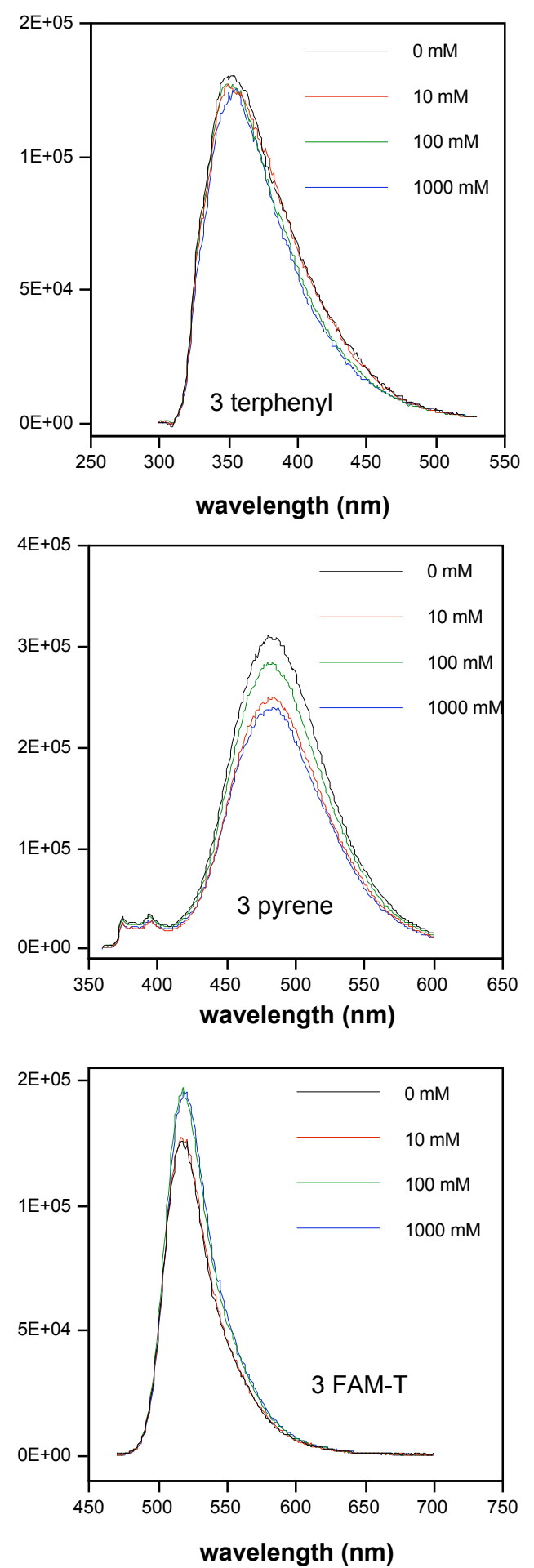

Fig. S3. Effects of ionic strength on the fluorescence of three trimeric fluorophores composed of terphenyl, pyrene, or fluorescein-T (FAM-T) as shown. NaCl concentrations are given. 

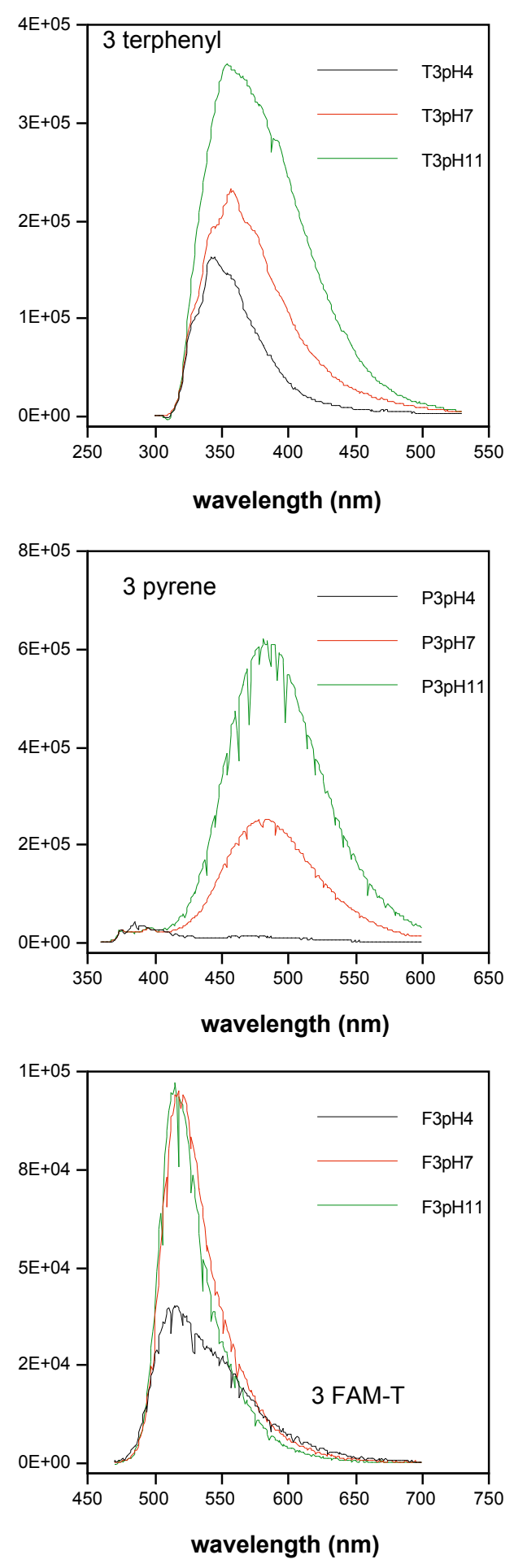

Fig. S4. Effects of pH on three trimeric fluorophores composed of terphenyl, pyrene, and FAM-T as shown above. 

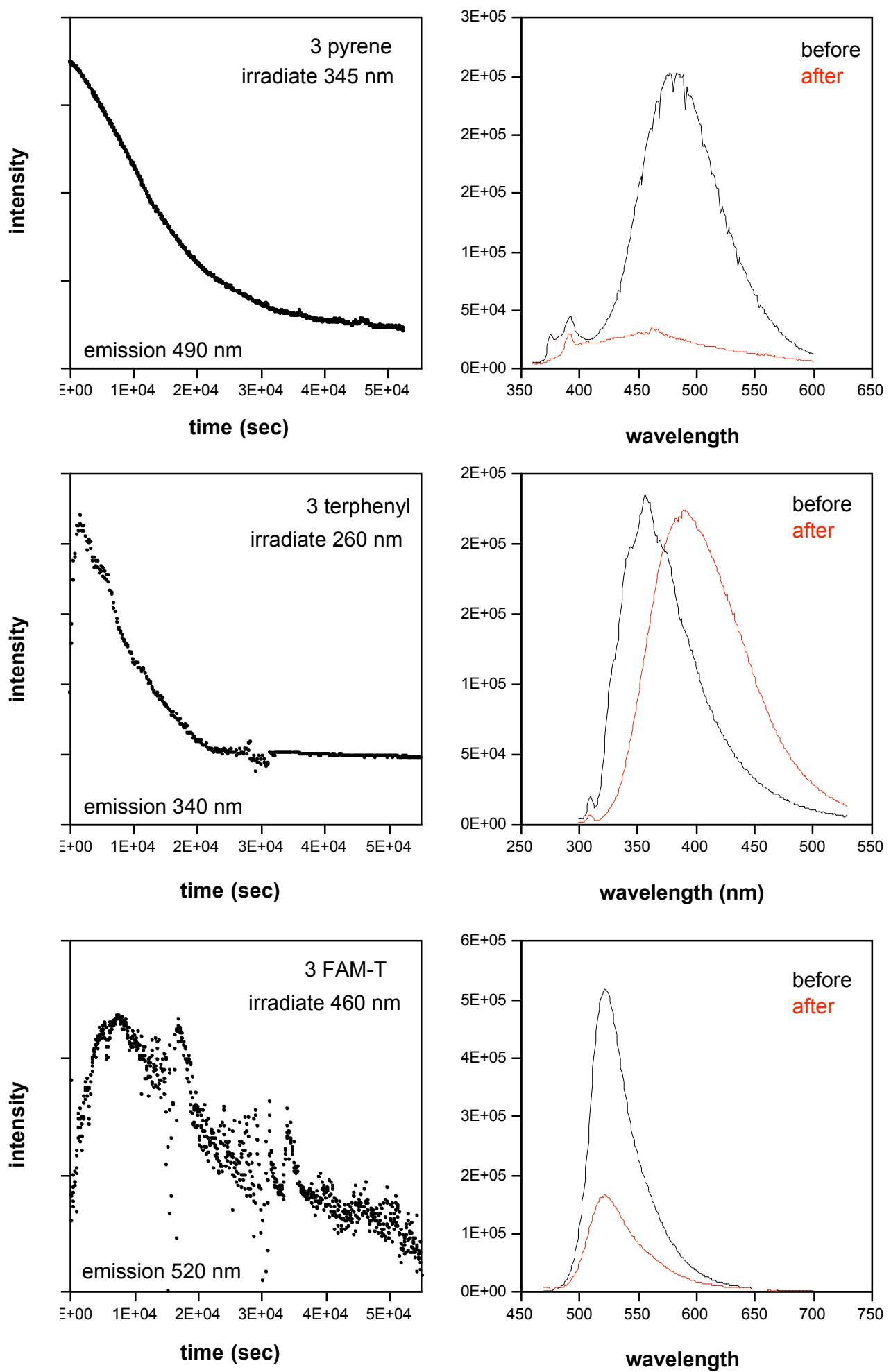

Fig. S5. Initial tests of photobleaching of oligomeric dyes. Dyes were irradiated in a SPEX fluorescence spectrometer with $20 \mathrm{~nm}$ slit widths centered at the absorption maxima (shown above), and were monitored over time (in air) at their emission maxima. Emission spectra before and after the irradation experiment are shown at right. Buffer contained $100 \mathrm{mM} \mathrm{NaCl}, 10 \mathrm{mM} \mathrm{MgCl} 2$, and $10 \mathrm{mM} \mathrm{Na} \cdot \mathrm{PIPES}, \mathrm{pH}=7$. 